\title{
Effects of Mental Practice on Function and Muscle Activation of Upper Extremity in Stroke Patients
}

\author{
Ju-Hyung Park, $\mathrm{OT}^{\dagger}$ \\ Department of Occupational Therapy, Kyungbuk College
}

Received: July 29, 2016 / Revised: July 31, 2016 / Accepted: August 7, 2016

(C) 2016 J Korean Soc Phys Med

\section{| Abstract |}

PURPOSE: The objective of this research is to investigate the effectiveness of mental practice on function and muscle activation of upper extremity (UE) in stroke patients.

METHODS: The mental practice was conducted on 12 subjects for 10 minutes and the related existing work treatment for 20 minutes. The evaluation for the function of UE proceeded with Fugl-Meyer assessment of motor function (FMA) and muscle activity analysis instrument. Furthermore we analyzed patients' reaching activity by two stages, reaching phase and returning to original position phase.

RESULTS: According to the research results, the subjects' upper limb function improved in all of the measured items and the total scores after the mental practice $(\mathrm{p}<.05)$. In muscle activity, CCR value was used to efficiently analyze the patients' reaching activity in the two stages of reaching stage and returning to the original position phase. While the elbow movement only had a bit of positive change (1.1\%) in the reaching stage, both the shoulder (-12.3\%) and elbow $(-18.2 \%)$ movements had a positive change in the returning

†Corresponding Author : juhyungi79@hanmail.net

This is an Open Access article distributed under the terms of the Creative Commons Attribution Non-Commercial License (http://creativecommons.org/licenses/by-nc/3.0) which permits unrestricted non-commercial use, distribution, and reproduction in any medium, provided the original work is properly cited. to original position phase.

CONCLUSION: This research confirmed that the mental practice is effective for the enhancement of UE function for stroke patients. The result of this research can contribute to the progress of mental practice in clinical environment.

Key Words: Mental Practice, Muscle Activity, Stroke, Upper Extremity Function

\section{Introduction}

Various therapeutic approaches are being introduced to increase the upper extremity (UE) function of stroke patients. General exercise therapy includes range of motion exercise, strength exercise, and aerobic exercise (Platz et al., 2005). The therapies based on the theory of brain plasticity and reeducation of the nervous system include constraint induced movement therapy, neuro muscular electrical stimulation, action observation, mirror therapy, and mental practice (Mangold et al., 2009).

The mental practice is a therapeutic method of training just by thinking about the assessment that is given without direct movement or stimulation of the outside (Jackson et al., 2004). According to the study of Deutsch and Newell (2004), the effect of mental practice brings out the change 
of the exercise-related area of the brain and increase in physical functions. However most studies used evaluation tools such as Fugle-Meyer Motor Assessment (FMA), Action Research Arm Test (ARAT), and Jebsen hand function test (Barclay-Goddard et al., 2011). These evaluation tools had limitations of evaluation without excluding the supervision of therapist even while assessing according to the trained evaluator for the qualitative evaluation of UE movement.

There are a variety of objective evaluation methods on mobility of hand and UE, but lately there have been attempts of a kinetic and analytic study by means of 3D motion analysis and electromyogram (Yoo et al., 2005). Electromyogram is a method which is used in a number of studies (Jun, 2011; Kim and Kang, 2013; Oh, 2015), detecting the electronic activity of skeletal muscles, a change in electric potential that follows innervation due to spontaneous tension and contraction of skeletal muscles after the reflection and voluntary movement (Hwang, 2006). The measurement data at electromyogram are used to analyze the quantity and pattern of muscle activity (Ma and Trombly, 2004). Therefore, this study makes verification through detailed analysis about the qualitative change of UE function by using muscle activity analysis equipment as well as FMA such as a clinical measurement.

\section{Methods}

\section{Experimental design and Participants}

This study was composed in one group pre-post test design. Research group were composed based on the selection criteria and exclusion criteria. Study subjects were selected from 12 stroke patients who participated in the rehabilitation program of D Hospital located in Seoul. The selection criteria included person that received stroke diagnosis for over 5 months, person that has over 24 points for Mini Mental State Examination-Korean (MMSE-K), person that has average score of below 2.26 in the Vividness of Movement Imagery Questionnaire (VMIQ), person that does not have hearing or sight disorders, and person that does not have aphasia based on chart review and the clinical observation of therapist. The exclusion criteria included person that showed pain in the affected side, unstable medical condition, depression based on chart review and the clinical observation of therapist, and patients below 2 of Brunnststrom Stage for arms and hands. The general characteristics of the subjects are noted in Table 1. The subjects received an explanation about the purpose and methods of the study prior to participation and provided informed consent according to the ethical principles of the Declaration of Helsinki.

Table 1. General characteristic of subjects $(\mathrm{N}=12)$

\begin{tabular}{lll}
\hline \multicolumn{1}{c}{ Variable } & \multicolumn{1}{c}{ Subject } & \multicolumn{1}{c}{ Ranges } \\
\hline Gender (Male/Female) & $6 / 6$ & none \\
Age (years) & $61.42 \pm 8.12^{*}$ & $54 \sim 73$ \\
Height $(\mathrm{cm})$ & $162.49 \pm 7.23^{*}$ & $150 \sim 178$ \\
Weight $(\mathrm{kg})$ & $65.85 \pm 7.23^{*}$ & $50 \sim 77$ \\
Stroke type & $6 / 6$ & none \\
(Ischemic/Hemorrhage) & & \\
Affected side (Lt/Rt) & $5 / 7$ & none \\
Month from stroke onset & $69.45 \pm 21.51^{*}$ & $42 \sim 94$ \\
MMSE-K & $24.66 \pm .77^{*}$ & $24 \sim 26$ \\
VMIQ & $1.98 \pm .13^{*}$ & $1.79 \sim 2.12$ \\
Brunnststrom Stage 3 6 & 12 & $3 \sim 6$ \\
\hline Lt: Left, Rt: Right, Mean \pm SD* $^{*}$ &
\end{tabular}

\section{Intervention}

Generally, mental practice refers to the training method of imagining while listening to the explanation of assessment movement (Dickstein, 2004). The non-paralysis movement was imagined first in the external aspect and then the movement of the paralysis part was imagined. Then the sense information to feel during the movement of non-paralysis part was imagined, and then the subject 
imagined the movement information that was the same in the paralysis part.

Mental practice was executed once a day, 5 times for 4 weeks, and total of 20 times for 200 minutes. The content of the mental practice was composed with 3 activities that can be approached in daily life. The 3 activities include: 1) bringing plastic cup that is on the table, 2) taking out small book from the drawer, and 3) wiping the desk with a towel.

\section{Outcome Measure}

1) Fugl-Meyer assessment of motor function (FMA)

FMA was used that evaluates the damage level and function level of stroke patients. This was used to evaluate the UE function of subjects in this study (Fugl-Meyer et al., 1975). This assessment criterion is classified into motor function, balance, sense, range of motion, and pain. From there, the motor function is classified into 64 points for upper limb and 34 points for lower limb totaling to 100 points. Also, balance 14 points, sense 24 points, range of motion 44 points, and pain 44 points are included where the total points for all items is 226 points. Each item must be scored from 0-3 points. This study assessed the UE motor function part. The assessment of each item was executed 3 times, and the highest score was checked. The reliability among measurements was $\mathrm{r}=.94$ and the reliability within measurements was r=.99 for Fugl-Meyer assessment of motor Function (U/E).

\section{2) sEMG (surface Electromyography)}

BTS FreeEMG 300 (BTS FreeEMG 300, BTS, Italy), a surface wireless electromyogram measuring system was used to measure muscle activation. In measuring the muscle electromyogram in relation to mobility of upper extremity, $\mathrm{S} / \mathrm{N}$ was $96 \mathrm{~dB}$ and CMRR (Common Mode Rejection Ratio) was $123 \mathrm{~dB}$, respectively. Four of the eight channels with the $1 \mathrm{KHz}$ sampling frequency were used to measure the hemi-side of deltoid anterior, deltoed posterior, biceps brachii, and triceps brachii of the upper extremity. The following parts of muscles were attached with electrodes to measure muscle activity levels. Deltoid anterior had electrodes attached at one finger width distal and anterior to the acromion. Next, Deltoid posterior had electrodes in the area about two finger widths posterior of the acromion. Biceps brachial had electrodes attached on the line between the medical acromion and the fossa cubit at 1/3 from the fossa cubit. Finally, for Triceps brachii, electrodes were attached at $50 \%$ on the line between the posterior crista of acromion and the olecranon at the olecranon at 2 finger widths medical to the line. Those whose raw data were automatically indicated by Myolab (Myolab, BTS, Italy) used in BTS FreeEMG 300 (BTS FreeEMG 300, BTS, Italy) were selectively processed. For the measurement of muscle activity, while the subject executed cup holding assessment, the data was collected. Holding cup in the original posture was set as stage 1, putting town the cup and going back to the original spot was set as stage 2 . The assessment was performed 3 times in total for before and after the experiment and the average value was used. The measured muscle activity signal was analyzed by using room mean square (RMS) method.

In addition, CCR (Co-contraction ratio) value was calculated based on RMS value measured before and after the intervention to analyze the coordination ability and improvement movement during the activity. The CCR value is calculated as the RMS value of antagonistic muscle on the RMS value of agonistic muscle, which is measured in the movement of designated sections of the activity. Also, the reduced CCR value after the intervention means an improved coordination and softer movement (Busse et al., 2005). The formula to calculate the CCR value is as follows (Fig. 1.). 


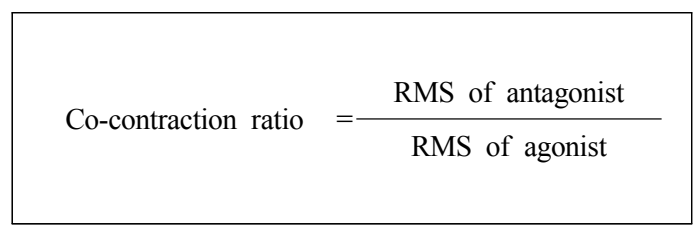

Fig. 1. The calculation of co-contraction ratio

\section{Statistical Analysis}

The statistical analysis of this study used PASW 18.0 and Excel program. Descriptive statistics was used to analyze the general characteristics of subjects, and paired samples t-test was used to measure the changes in the UE functions before and after the intervention. The CCR value through muscle activity was calculated using the Excel program.

\section{Results}

\section{Comparison of upper extremity function between} pre and post-test

According to the comparison of upper extremity functions before and after the intervention, the UE functions had a significant improvement $(\mathrm{p}<.05)$ as the total points of FMA (U/E) increased from $41.50 \pm 12.39$ to $48.70 \pm 11.03$. There were significant improvements respectively in all detailed items investigated $(\mathrm{p}<.05)$ (Table 2$)$.
Table 2. Comparison of Upper Functions

$(\mathrm{N}=12)$

\begin{tabular}{cccccc}
\hline & & pre & post & & \\
& & Mean \pm SD & Mean \pm SD & t & p \\
\hline \multirow{2}{*}{ FMA } & shoulder & $24.40 \pm 7.48$ & $28.60 \pm 6.97$ & -2.849 & $.019^{*}$ \\
& wrist & $6.70 \pm 2.62$ & $8.00 \pm 2.26$ & -2.414 & $.039^{*}$ \\
& hand & $10.40 \pm 3.13$ & $12.10 \pm 2.28$ & -2.847 & $.019^{*}$ \\
& total & $41.50 \pm 12.39$ & $48.70 \pm 11.03$ & -2.935 & $.017^{*}$ \\
\hline
\end{tabular}

FMA : Fugl-Meyer assessment of motor Function, $\mathrm{p}<.05^{*}, \mathrm{p}<.01^{* *}$

2. Comparison of co-contraction ratio between pre and post-test

When the CCR value at reaching before and after the intervention was compared, shoulder flexion was .42 before intervention and .48 after intervention, showing 14.2 as the change of CCR value, while elbow reaching was 1.92 before intervention and 1.90 after intervention, showing the change of -1.1. This shows there was a bit of negative change in shoulders but a bit of positive change in elbows (Table 3). When the CCR value at position returning before and after the intervention was compared, shoulder extension was 1.88 before intervention and 1.65 after intervention, showing -12.3 as the change of CCR value, while elbow flexion was .44 before intervention and .36 after intervention, showing the change of -18.2. This shows there positive changes in both shoulders and elbows (Table 3).

Table 3. Comparison of co-contraction ration between pre and post-test

\begin{tabular}{ccccc}
\hline \multirow{2}{*}{ Stage } & CCR & $\begin{array}{c}\text { pre } \\
\text { Mean } \pm \text { SD }\end{array}$ & $\begin{array}{c}\text { post } \\
\text { Mean } \pm \text { SD }\end{array}$ & Percent change \\
\hline \multirow{2}{*}{ Reaching stage } & Shoulder Flexion [DP/DA] & $.42 \pm .15$ & $.48 \pm .40$ & 14.2 \\
& Elbow Extension [BI/TRI] & $1.92 \pm 1.10$ & $1.90 \pm .66$ & -1.1 \\
\hline $\begin{array}{c}\text { Returning to original } \\
\text { position phase }\end{array}$ & Shoulder Extension [DA/DP] & $1.88 \pm .89$ & $1.65 \pm 1.19$ & -12.3 \\
\hline
\end{tabular}

CCR : Co-contraction ratio, DA : Deltoid Anterior, DP : Deltoid Posterior, BI : Biceps Brachii, TRI : Triceps Brachii 


\section{Discussion}

This research analyzed the changes of Fugl-Meyer Assessment and the changes of muscle activity before and after intervention to observe the improvement of upper extremity functions. This research has a significant change in all areas of upper extremity that were observed, which corresponded to the results of research that previously applied mental practice to improve upper extremity functions (Page et al., 2001; Page et al., 2007). Many other studies that applied the mental practice on stroke patients and verified its effectiveness through Fugl-Meyer Assessment had the same result (Kim et al., 2010).

According to the previous studies that applied mental practice on existing stroke patients, most tools to evaluate the measurement could not exclude the subjectivity of therapist although they were used by trained evaluators to assess the UE movement qualitatively (Kim et al., 2010; Barclay-Goddard et al., 2011). To measure the muscle activity of specific muscles during the actual patients' UE movement, it used BTS Free EMG 300 (BTS Free EMG 300, BTS, Italy), a wireless surface EMG. With this tool the evaluators measured the patients' movement of putting a plastic cup on a place that was far away as one's arm length, reaching hands to grab it and putting it down, while seated on the table. Anterior deltoid, posterior deltoid, biceps brachii, and triceps brachii, which were suggested as the appropriate muscles by study of Molteni et al. (2008), were selected to measure the stroke patients' upper extremity functions.

Data was collected over two phases: the first phase was grabbing the cup from the original position, and the second phase was putting the cup down and returning to the original position. The CCR (Co-contraction ratio) value of subjects before and after the intervention was measured to verify the coordination capability and qualitative movement of stroke patients' upper extremity functions through the actual mental practice. In the mental practice group, the patients had a negative change in the first phase, reaching activity as there was a positive value of $14.2 \%$ for shoulder movement after intervention, while there was a bit of positive change after the intervention as the change of elbow movement had a negative value of $-1.1 \%$. As for the CCR value when returning to the original position after reaching arms in the second phase, the subjects' shoulder (-12.3\%) and elbow (-18.2\%) movement had negative values of change after the intervention, indicating that there was a positive change.

Such results corresponded to the results of Cha et al. (2015)'s research, which was a case study that applied mental practice on the existing stroke patients to improve their UE functions and measured their muscle activity. In this study all three participating subjects had an increase in muscle activity, and the changes of CCR value were $-24.7 \%,-34.9 \%$, and -21.3 after intervention, showing that mental practice improved the coordination capability of the stroke patients' UE functions and their movement more qualitatively. This supports many study results that mentioned stroke patients' UE functions through mental practice, although they did not performed measurement and analysis through the existing muscle activity (Kim et al., 2010; Barclay-Goddard et al., 2011).

The limitations of this research are as follows. First, it was difficult to directly compare with many other study results due to the insufficient amount of studies that applied mental practice and measured muscle activity to improve the UE functions of stroke patients. In addition, this research could not check the direct changes of brain because it did not use a brain imaging technique such as fMRI or PET, and lacked a bit in the verification of effectiveness depending on the level of UE functions of patients when applying mental practice. Also, it was difficult to generalize the results of this research because it was a single-group pretest-posttest study without a control group. This study will be more meaningful in the clinical setting of domestic occupational therapy if it improves these limitations in the future. 


\section{Conclusion}

The objective of this research is to verify the effectiveness of mental practice in an objective manner. In conclusion, this research confirmed that the mental practice is effective for the enhancement of UE function for stroke patients. The result of this research can contribute to the progress of mental practice in Korean clinical environment. Further researches on developing more versatile programs in a form of mental practice would be necessary to expand this research.

\section{References}

Barclay-Goddard RE, Stevenson TJ, Poluha W, et al. Mental practice for treating upper extremity deficit in individuals with hemiparesis after stroke (Review). Cochrane Database Syst Rev. 2011;11(5).

Busse ME, Wiles CM, van Deursen RW, et al. Muscle co-activation in neurological conditions. Phys Ther Rev. 2005;10:247-53.

Cha YJ, Yoo EY, Jung MY, et al. Effects of mental practice with action observation training on occupational performance after stroke. J Stroke Cerebrovasc Dis. 2015;24(6):1405-13.

Deutsch KM, Newell KM. Changes in the structure of children's isometric force variability with practice. J Exp Child Psychol. 2004;88:319-33.

Dickstein R, Dunsky A, Marcovitz E, et al. Motor imagery for gait rehabilitation in post-stroke hemiparesis. Phys Ther. 2004;84(12):1117-77.

Fugl-Meyer AR, Jääskö L, Leyman I, et al. The post-stroke hemiplegic patient. 1. a method for evaluation of physical performance. Scand J Rehabil Med. 1975; 7(1):13-31.

Hewett TE, Ford KR, Levine P, et al. Reaching kinematics to measure motor changes after mental practice in stroke. Top Stroke Rehabil. 2007;14(4):23-9.

Hwang KY. EMG analysis upper extremity by satbar-grip patterns of ssirum (Korea traditional wrestling) Master's Degree. Chung-Ang University. 2006.

Jackson PL, Doyon J, Richards CL, et al. The efficacy of combined physical and mental practice in the learning of a foot-sequence task after stroke: a case report. Neurorehabil Neural Repair. 2004;18(2):106-11.

Jun SM. Comparison of Electromyography Muscle in Lower Extremity from Isometric Trunk Exercise for 12weeks of Elderly Hansen's disease in Facility-Dwelled and Foot Sensory Loss. J Korean socphys med. 2011; 6(1):93-101.

Kim CY, Kang JH. Analysis of Electromyographic Activities of Erect Spinae at Different Height of Table during Ultrasound Therapy Work. J Korean socphys med. 2013;8(3):289-94.

Kim K, Kim JS, Oh DW, et al. Clinical effectiveness of lower extremity and upper extremity performance on mental practice for adult with post stroke hemiplegia: a systematic review. Journal of Special Education \& Rehabilitation Science. 2010;49(4):201-20.

Kwon YD, Chang HJ, Choi YJ, et al. Nationwide trends in stroke hospitalization over the past decade, J Korean Med Assoc. 2012;55(10):1014-25.

Lee JH, Yum DS. The application of the new health insurance fee establishment on the occupational therapy service: A Case Study. J Korean Soc Occup Ther. 2006;14(3):85-92.

Ma $\mathrm{H}$, Trombly CA. Effects of task complexity on reaction time and movement kinematics in elderly people. Am J Occup Ther. 2004;58(2):150-8.

Mangold S, Schuster C, Keller T, et al. Motor training of upper extremity with functional electrical stimulation in early stroke rehabilitation. Neurorehabil Neural Repair. 2009;23(2):184-90.

Molteni F, Caimmi A, Cazzaniga A, et al. Using surface dynamic electromyography during upper extremity robotic 
training. Europa Medicaphysica. 2008;44(3):1-3.

Muller K, Butefisch CM, Seitz RJ, et al. Mental practice improves hand function after hemiparetic stroke. Restor Neurol Neurosci. 2007;25(5):501-11.

Oh DW. Influences of Shoe Heel Height on Isometric Shoulder Abductor Strength and EMG Activities of Selected Shoulder Muscles. J Korean socphys med. 2015;10(2):9-16.

Page SJ, Levine P, Sisto SA, et al. Mental practice combined with physical practice for upper-limb motor deficit in subacute stroke. Phys Ther. 2001;81(8):1455-62.

Page SJ, Levine P, Leonard A, et al. Mental practice in chronic stroke. Stroke. 2007;38:1293-97.
Platz T, Eickhof C, Van-Kaick S, et al. Impairment-oriented training or Bobath therapy for severe arm paresis after stroke: A single-blind, multicentre randomized controlled trial. Clinic Rehab. 2005;19(7):714-24.

Riccio I, Iolascon G, Barillari MR, et al. Mental practice is effective in upper limb recovery after stroke: a randomized single-blind cross-over study. Eur J Phys Rehabil Med. 2010;46(1):19-25.

Yoo WG, Park JH, Kim MH, et al. Velocity of reaching and vertical displacement during various bimanual reaching target activities. J Korean Soc Occup Ther. 2005;13(2):41-9. 